\title{
Measures of quantum computing speedup
}

\author{
Anargyros Papageorgiou, 1 , J and Joseph F. Traub ${ }^{1, \oplus}$ \\ ${ }^{1}$ Department of Computer Science, Columbia University, New York, NY 10027, USA.
}

\begin{abstract}
We introduce the concept of strong quantum speedup. We prove that approximating the ground state energy of an instance of the time-independent Schrödinger equation, with $d$ degrees of freedom, $d$ large, enjoys strong exponential quantum speedup. It can be easily solved on a quantum computer. Some researchers in discrete complexity theory believe that quantum computation is not effective for eigenvalue problems. One of our goals in this paper is to explain this dissonance.
\end{abstract}

PACS numbers: 03.67.Ac, 02.60.-x, 02.70.-c

\section{QUANTUM SPEEDUP CRITERIA FOR COMPUTATIONAL SCIENCE}

How much faster are quantum computers than classical computers for important problems? We shall show that the answers depend critically on how quantum speedup is measured.

We begin with two criteria for quantum speedup. Criterion 1. For a given problem, the quantum speedup, $S_{1}$, is the ratio between the cost of the best classical algorithm known and the cost of a quantum algorithm. That is

$$
S_{1}=\frac{\text { cost of the best classical algorithm known }}{\text { cost of a quantum algorithm }} .
$$

The cost of an algorithm is the amount of resources it uses, such as the number of arithmetic operations, or elementary quantum operations, the number of oracle calls, etc.

Our second criterion uses the concept of computational complexity which, for brevity, we will call complexity. By complexity we mean the minimal cost of solving a problem.

Criterion 2. For a given problem, the strong quantum speedup, $S_{2}$, is the ratio between classical complexity and quantum complexity. That is,

$$
S_{2}=\frac{\text { classical complexity }}{\text { quantum complexity }}
$$

We are particularly interested in finding problems where $S_{1}$ or $S_{2}$ are exponential functions of a parameter. We then refer to exponential quantum speedup and strong exponential quantum speedup, respectively. The crucial difference between the two criteria is that if a problem satisfies Criterion 1 someone may invent a better classical algorithm and therefore decrease $S_{1}$ but if a problem satisfies Criterion 2 then $S_{2}$ cannot be decreased.

We apply these criteria to a well-known problem. Interest in quantum computing received a major boost in

*Electronic address: ap@cs.columbia.edu

†Electronic address: traub@cs.columbia.edu
1994 [1] when Peter Shor showed that factoring a large integer, $N$, can be done with cost polylog in $N$. The best classical algorithm known, the general number field sieve, has super-polynomial cost

$$
O\left(2^{c(\log N)^{1 / 3}(\log \log N)^{2 / 3}}\right) .
$$

Thus integer factorization has super-polynomial quantum speedup. However, it is not known to have strong super-polynomial quantum speedup. Although deciding whether an integer is prime can be done with polynomial cost [2], it is still an open problem to determine whether there exists an efficient algorithm for computing a nontrivial factor of a composite integer. This is a problem in the complexity class FNP, which is a functional analogue of the class NP for decision problems; see [3]. At the moment it is only conjectured that there is no polynomial factoring classical algorithm; it is possible that some day a polynomial-cost factorization algorithm will be found.

Often scientists prove exponential quantum speedup for a problem but not strong exponential quantum speedup because the classical complexity is not known. Examples include [4-10]. Establishing exponential quantum speedups marks an important advance in understanding the power of a quantum computer.

We discuss three problems which enjoy strong quantum speedups. We emphasize that we do not claim separations of the complexity hierarchy in the sense $P \neq N P$. Separation questions lead to open problems which are believed to be very hard. Our complexity estimates and speedups are obtained using specific kinds of oracle calls.

The first problem is integration of functions of $d$ variables, which have uniformly bounded first partial derivatives. This is a typical continuous problem in computational science. Since such problems have to be discretized we have only partial information in the computer about the mathematical integrand. We obtain this partial information using oracles, for example, function evaluations.

The cost of an algorithm is equal to the number of oracle calls plus the number of operations the algorithm uses to combine the values returned by the oracle calls to obtain the approximate answer. In this sense, the oracle calls can be viewed as the input to the algorithm and the combinatory cost is a function of the number of oracle calls. 
The problem complexity is the minimal cost of any algorithm for solving the problem to within error $\varepsilon$. Let $m(\varepsilon, d)$ be the number of oracle calls for solving the problem. This is called the information complexity. Clearly the information complexity is a lower bound on the complexity. This lower bound does not require knowledge of the combinatory cost of an algorithm. For our integration problem $m(\varepsilon, d)$ is of order $\varepsilon^{-d}$. Thus the problem suffers the curse of dimensionality on a classical computer.

On a quantum computer the cost of the algorithm is the number of queries and other operations required to solve the problem to within $\varepsilon$ with probability greater than $1 / 2$. As before, the problem complexity is the minimal cost of any algorithm. The query complexity, i.e., the minimum number of quantum queries, provides a lower bound on the problem complexity. For our example of multivariate integration the quantum complexity is $o\left(\varepsilon^{-2}\right)[11,12]$. The curse of dimensionality is vanquished by a quantum computer. This problem enjoys strong exponential quantum speedup.

The second problem is a discrete example which also uses oracle calls. The problem is to compute the mean of a Boolean function, $f$, of $n$ variables to within error $\varepsilon$. The minimal number of evaluations of $f$, i.e., the number of oracle calls, is $2^{n-1}(1-\varepsilon)$ in the worst case. The amplitude estimation algorithm of Brassard et al. 13] can be used to compute the mean with $O\left(\varepsilon^{-1}\right)$ queries, for all $n \gg \log \varepsilon^{-1}$, plus a polynomial in $n$ and $\log \varepsilon^{-1}$ number of quantum operations that are required for the remaining parts of the algorithm excluding the queries. Thus this problem also enjoys strong exponential quantum speedup.

Finally, we mention that Simon's problem 14 is solved using quantum queries and enjoys a strong exponential quantum speedup.

In the next section we will analyze the quantum speedup of approximating the ground state energy of the time-independent Schrödinger equation under certain conditions on the potential $V$. We will argue that this problem also enjoys strong exponential quantum speedup. Some researchers in discrete complexity theory believe that quantum computation is not effective for eigenvalue problems. We will try to resolve this dissonance.

\section{COMPUTING THE GROUND STATE ENERGY}

Consider the time-independent Schrödinger equation

$$
\begin{array}{cl}
-\Delta u(x)+V(x) u(x)=\lambda u(x) & x \in I_{d}:=(0,1)^{d} \\
u(x)=0 & x \in \partial I_{d} .
\end{array}
$$

Thus the equation is defined on the unit cube in $d$ dimensions with a Dirichlet boundary condition. As usual,

$$
\Delta=\sum_{j=1}^{d} \frac{\partial^{2}}{\partial x_{j}^{2}}
$$

denotes the Laplacian operator and $V \geq 0$ is the potential. Assume $V$ is uniformly bounded by unity, i.e., $\|V\|_{\infty} \leq 1$, that it is continuous and has continuous first partial derivatives $D_{j} V:=\partial V / \partial x_{j}, j=1, \ldots, d$, which satisfy $\left\|D_{j} V\right\|_{\infty} \leq 1$. The problem is to approximate the ground state energy (i.e, the smallest eigenvalue) with relative error $\varepsilon$, using function evaluations of $V$.

Using perturbation arguments it was shown in [15] that the ground state energy approximation is at least as hard as multivariate integration for $V$ satisfying the conditions above. Using lower bounds for multivariate integration [16] we conclude that on a classical computer with a worst case guarantee the complexity is at least proportional to

$$
(c d \varepsilon)^{-d} \quad \text { as } d \varepsilon \rightarrow 0,
$$

where $c>1$ is a constant. In fact, this lower bound follows from the number of evaluations of $V$ (oracle calls) that are necessary for accuracy $\varepsilon$. It is assumed that $V$ is known only at the evaluation points. The complexity is an exponential function of $d$. Moreover, discretizing the partial differential operator on a regular grid with mesh size $\varepsilon$ leads to a matrix eigenvalue problem, where the matrix has size $\varepsilon^{-d} \times \varepsilon^{-d}$. The evaluations of $V$ appear in the diagonal entries. Inverse iteration can be used to approximate the minimum eigenvalue with a number of steps proportional to $d \log \varepsilon^{-1}$.

The cost on a quantum computer is of order

$$
d \varepsilon^{-(3+\delta)} \text { for any } \delta>0,
$$

and the number of qubits is proportional to

$$
d \log \varepsilon^{-1} .
$$

The algorithm uses queries returning evaluations of $V$ truncated to $O\left(\log \varepsilon^{-1}\right)$ bits. Its cost includes the number of queries and quantum operations. See [17] for details. We remark that for a number of potentials $V$, such as the ones given by polynomials or trigonometric functions satisfying our assumptions, the queries can be implemented by quantum circuits of size polynomial in $d \log \varepsilon^{-1}$. The cost of the quantum algorithm provides an upper bound for the complexity. A lower bound of order $(d \varepsilon)^{-1 / 2}$ for the quantum complexity follows by considering $V$ to be the sum of $d$ univariate functions. From the upper and lower quantum complexity bounds one obtains a range for the anticipated speedup. Recall that the criterion for strong quantum speedup is

$$
S_{2}=\frac{\text { classical complexity }}{\text { quantum complexity }}
$$

Hence,

$$
\begin{aligned}
& S_{2}=O\left(\frac{(c d \varepsilon)^{-d}}{(d \varepsilon)^{-1 / 2}}\right) \\
& S_{2}=\Omega\left(\frac{(c d \varepsilon)^{-d}}{d \varepsilon^{-(3+\delta)}}\right) \cdot \text { as } d \varepsilon \rightarrow 0 .
\end{aligned}
$$


Thus quantum computation enjoys strong exponential quantum speedup, in $d$, over the worst case deterministic classical computation.

Another way of describing this is to say that quantum computation vanquishes the curse of (exponential) dimensionality. The reason for the word in parentheses is that Richard Bellman coined the phrase curse of dimensionality informally in the preface of [18] in the study of dynamic programming for the solution of optimization problems. He noted that as the number of state variables increases solving the problem gets harder. This was an empirical result since it was before computational complexity theory was created. He did not specify how the difficulty of the problem depended on the number of state variables which is why we inserted the word exponential.

\section{A. Comparison with QMA-complete problems}

Several researchers have written us (private communications) that strong exponential quantum speedup for the ground state energy problem cannot be true because it would imply that we have established complexity class separations. We do not claim such separation results.

To illustrate this issue we will describe the assumptions appropriate for the continuous problems of computational science comparing them to those for discrete problems.

We begin with the computational complexity of continuous problems. This is studied in the field of informationbased complexity. There are numerous monographs and surveys describing the foundations and results. See, for example, [16, 19 26].

Typically, these problems are solved numerically and therefore approximately to within error $\varepsilon$. In applications the user chooses the appropriate $\varepsilon$. Another key parameter is $d$ which denotes the degrees of freedom or dimension of the problem. The size of the input, the algorithm cost and the problem complexity depend on $\varepsilon$ and $d$.

We specialize to the ground state energy problem. The continuous problem has structure which can be exploited by the quantum algorithm. The Hamiltonian is $-\frac{1}{2} \Delta+V$, where $\Delta$ is the Laplacian and $V$ is the potential. In the previous section we specified the conditions on $V$.

After discretization the resulting matrix is the sum of two matrices. One involves the discretized Laplacian $\Delta_{\varepsilon}$ while the other is a diagonal matrix $V_{\varepsilon}$ which contains function evaluations of the potential $V$. All the properties of $\Delta$ and $\Delta_{\varepsilon}$ are well known as well as the relationship between them. This includes the eigenvalues, their separation, their distribution, the eigenvectors etc. 2729]. In particular, for small $\varepsilon$ the smallest eigenvalue of $-\frac{1}{2} \Delta+V$ is close to that of $-\frac{1}{2} \Delta_{\varepsilon}+V_{\varepsilon}$. The discretization has preserved the problem structure. Most importantly, since the norm of $V_{\varepsilon}$ is small the eigenvalues of $-\frac{1}{2} \Delta_{\varepsilon}+V_{\varepsilon}$ are close to those of $-\frac{1}{2} \Delta_{\varepsilon}$.

Turning to discrete problems, the complexity class
QMA is the quantum analogue of NP. It is also called BQNP and the name QMA was given by Watrous [30]. A decision problem about the ground state energy of local Hamiltonians is a QMA-complete problem 31 33] and this has been a very important and influential result in discrete complexity theory. Hence, it is believed it is very hard to solve. A number of papers state that approximating the ground state energy is a QMA-hard problem; see, e.g., 34, 35]. A similar conclusion holds for a density functional theory approach for eigenvalue approximation 36].

There are important differences between our ground state problem and the local Hamiltonian QMA-complete problem. The deterministic worst case complexity lower bound of equation (1) has been obtained using an oracle and cannot provide a lower bound for the complexity of the QMA-complete local Hamiltonian problem whose input is provided explicitly. For the same reason it cannot imply a separation in the complexity hierarchy. Furthermore, it is unlikely that the quantum algorithm of [17] could be used to solve the local Hamiltonian problem. This algorithm was designed specifically to approximate the smallest eigenvalue of $-\frac{1}{2} \Delta_{\varepsilon}+V_{\varepsilon}$. Using it for the local Hamiltonian problem would require one to convert efficiently a sum of polynomially many local Hamiltonians to the sum of two matrices, where we know everything about the first and the second is a diagonal matrix with relatively small norm. Observe that in the case of the local Hamiltonian problem the input is a polynomial number of local Hamiltonians all satisfying the same properties [32, Def. 2.3] without any apparent useful distinction between them. While it is easy to deal with each Hamiltonian individually, dealing with their sum is a hard problem.

Moreover, the qubit complexity of our ground state problem is proportional to $d \log \varepsilon^{-1}$ and so is the number of qubits used by the quantum algorithm. The number of qubits for the QMA complete local Hamiltonian problem is denoted by $n$ in [32, Def. 2.3] and the sum of the local Hamiltonians is a $2^{n} \times 2^{n}$ matrix. Note that in the former case the number of qubits is derived from the parameters $\varepsilon$ and $d$, while in the latter case $n$ is the parameter. For the QMA-complete problem it is not known if there exists a quantum algorithm solving it with cost polynomial in $n$.

Relating the cost of the quantum algorithm in [17] to the number of its qubits highlights further differences between the cost requirements of discrete complexity theory and the continuous problems of physical sciences and engineering. We have shown that the ground state energy can be approximated with cost polynomial in $d$ and $\varepsilon^{-1}$ which makes it "easy". Yet from the point of view of discrete complexity theory such problems are considered hard. We believe discrete complexity theory sets too high a bar. It requires that the cost of the quantum algorithm is a polynomial in the number of qubits (or, equivalently, polylogarithmic in the matrix size) for the algorithm to be considered efficient (recall that in [32, Def. 2.3] $n$ is 
the number of qubits upon which each Hamiltonian acts, the Hamiltonian is a $2^{n} \times 2^{n}$ matrix, and the input size is polynomial in $n$.) See also [37].

Here is the crux of why the bar is too high for the continuous problems of computational science. With the exception of problems such as the solution of nonlinear equations and convex optimization, scientific problems cannot be solved with cost polynomial in $\log \varepsilon^{-1}$ [16]. One of the simplest continuous scientific problems is approximating a univariate integral with the promise that the integrand has a uniformly bounded first derivative. The classical complexity of this integration problem is $\Theta\left(\varepsilon^{-1}\right)$ and the quantum complexity is $\Theta\left(\varepsilon^{-1 / 2}\right)$; see [11, 38] for more details. As we stated earlier the lower bound for the quantum complexity of the ground state problem is proportional to $(d \varepsilon)^{-1 / 2}$. Note that in computational science $\varepsilon$ is often not very small. A typical value is $\varepsilon=10^{-8}$. Thus complexity of order $\varepsilon^{-1}$ or $\varepsilon^{-1 / 2}$ is not expensive. Note that the complexity of univariate integration is exponentially greater than $\log \varepsilon^{-1}$.

We have been asked by researchers in discrete complexity theory what if $\varepsilon$ is constant. As stated above $\varepsilon$ and $d$ are parameters and one studies the complexity for all their values. Secondly, this possibility is excluded for our ground state energy problem since then the problem is trivial. Indeed, unless $\varepsilon<1 / d$ the problem can be solved with constant cost by the algorithm that does not make any evaluations at all and returns the value $d \pi^{2}+1 / 2$, since then the error is bounded by $1 / d$.

\section{DISCUSSION}

If one looks at the approximation of the ground state energy of the $d$-dimensional Schrödinger equation through the prism of discrete complexity theory one would conclude that this problem is hard to solve on a quantum computer. We propose that the speedup measures $S_{1}$ or $S_{2}$ are more relevant for the power of quantum computing for problems in computational science.

We suggest that analogous results might be found if one were to investigate other continuous problems. That is they are tractable on a quantum computer from the point of view of computational science but intractable from the view of discrete complexity theory.

\section{Acknowledgements}

This work has been supported in part by NSF/DMS. We thank M. Yannakakis for his recommendation to highlight the differences in the models of computation.
[1] P. W. Shor, Proceedings of the 35th Annual Symposium on Foundations of Computer Science, IEEE Computer Society Press, Los Alamitos, CA pp. 124-134 (1994), arXiv.org/abs/quant-ph/9508027.

[2] M. Agrawal, N. Kayal, and N. Saxena, Annals of Mathematics 160(2), 781 (2004).

[3] C. H. Papadimitriou, Computational Complexity (Addison-Wesley, Reading, MA, 1994).

[4] D. S. Abrams and S. Lloyd, Phys. Rev. Lett. 83, 5162 (1999).

[5] A. Aspuru-Guzik, A. D. Dutoi, P. J. Love, and M. Head-Gordon, Science 309, 1704 (2005), URL http://www.sciencemag.org/cgi/content/abstract/309/

[6] S. P. Jordan, K. S. M. Lee, and J. Preskill, Quantum algorithms for quantum field theories (2011), http://arxiv.org/abs/1111.3633.

[7] P. Jaksch and A. Papageorgiou, Phys. Rev. Lett. 91, 257902 (2003).

[8] D. A. Lidar and H. Wang, Phys. Rev. E 59, 2429 (1999).

[9] H. Wang, S. Kais, A. Aspuru-Guzik, and M. Hoffmann, Phys. Chem. Chem. Phys. 10, 5388 (2008).

[10] J. Whitfield, J. Biamonte, and A. Aspuru-Guzik, Molecular Physics 109(5), 735 (2011).

[11] E. Novak, J. Complexity 19(1), 19 (2001).

[12] S. Heinrich, J. Complexity 19, 19 (2003).

[13] G. Brassard, P. Høyer, M. Mosca, and A. Tapp, Quantum Amplitude Amplification and Estimation (in Contemporary Mathematics, Quantum Computation and Information, Samuel J. Lomonaco Jr. and Howard E. Brandt, Editors, AMS, Providence, RI, 2002), vol. 305, p. 53, also http://arXiv.org/abs//quant-ph/0005055.

[14] D. R. Simon, SIAM J. Comput. 26, 1474 (1997).

[15] A. Papageorgiou, J. Complexity 23(4-6), 802 (2007).

[16] N. Novak and H. Woźniakowski, Tractability of Multivariate Problems, Volume I: Linear Information (European Mathematical Society, Zurich, 2008).

[17] A. Papageorgiou, I. Petras, J. F. Traub, and C. Zhang, Math. Comp. 82, 2293 (2013), also http://arxiv.org/abs/1008.4294.

[18] R. Bellman, Dynamic Programming (Princeton University Press, Princeton, NJ, 1957).

[19] J. F. Traub and H. Woźniakowski, A general theory of 5741/\$p̈0inal algorithms (ACM Monograph Series, Academic Press, New York, 1980).

[20] E. Novak, Deterministic and Stochastic Error Bounds in Numerical Analysis (Lecture Notes in Mathematics 1349, Springer-Verlag, Berlin, 1988).

[21] J. F. Traub, G. Wasilkowski, and H. Woźniakowski, Information-Based Complexity (Academic Press, New York, 1988).

[22] L. Plaskota, Noisy Information and Computational Complexity (Cambridge University Press, Cambridge, UK, 1996).

[23] J. F. Traub and A. G. Werschulz, Complexity and Information (Cambridge University Press, Cambridge, UK, 1998).

[24] E. Novak, I. H. Sloan, J. F. Traub, and H. Woźniakowski, Essays on the Complexity of Continuous Problems (European Mathematical Society, Zurich, 2009).

[25] N. Novak and H. Woźniakowski, Tractability of Multivari- 
ate Problems, Volume II: Standard Information for Functionals (European Mathematical Society, Zurich, 2010).

[26] N. Novak and H. Woźniakowski, Tractability of Multivariate Problems, Volume III: Standard Information for Operators (European Mathematical Society, Zurich, 2012).

[27] R. Courant and D. Hilbert, Methods of Mathematical Physics, Vol. I (Wiley Classics Library, WileyInterscience, New York, 1989).

[28] G. E. Forsythe and W. R. Wasow, Finite-Difference Methods for Partial Differential Equations (Dover, New York, 2004).

[29] E. C. Titschmarsh, Eigenfunction Expansions Associated with Second-Order Differential Equations, Part B (Oxford University Press, Oxford, UK, 1958).

[30] J. Watrous, Proc. 41st Annual IEEE Symposium on Foundations of Computer Science pp. 537-546 (2000).

[31] A. Kitaev, A. Shen, and M.Vyalyi, Classical and quantum computation (Grad. Stud. Math. Amer. Math. Soc.,
Providence, RI, 2002).

[32] J. Kempe, A. Kitaev, and O. Regev, SIAM J. Computing 35(5), 1070 (2006).

[33] D. Aharonov and T. Naveh, Quantum NP - A survey (2002), http://arxiv.org/abs/quant-ph/0210077.

[34] S. P. Jordan, D. Gosset, and P. J. Love, Phys. Rev. A 81(3), 032331 (2010).

[35] T.-C. Wei, M. Mosca, and A. Nayak, Phys. Rev. Lett. 104, 040501 (2010).

[36] N. Schuch and F. Verstraete, Nature Physics 5, 732 (2009).

[37] K. R. Brown, R. J. Clark, and I. L. Chuang, Phys. Rev. Lett. 97(5), 050504 (2006), also http://arXiv.org/abs/quant-ph/0601021.

[38] S. Heinrich and E. Novak, in Monte Carlo and QuasiMonte Carlo Methods 2000, K.-T. Fang, F. J. Hickernell and H. Niederreiter eds., Springer-Verlag, Berlin (2002). 